\title{
TEG in the monitoring of coagulation changes in patients with sepsis and the clinical significance
}

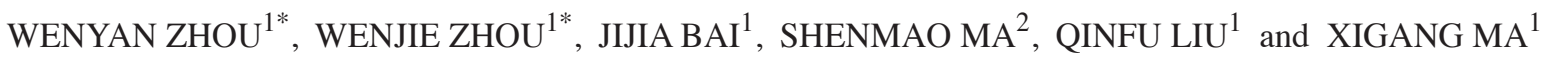 \\ ${ }^{1}$ Department of Intensive Care Unit and ${ }^{2}$ Resident Standardized Training Base, \\ General Hospital of Ningxia Medical University, Yinchuan, Ningxia 750004, P.R. China
}

Received June 11, 2018; Accepted January 22, 2019

DOI: $10.3892 /$ etm.2019.7342

\begin{abstract}
Application values of thromboelastography (TEG) in dynamic monitoring of coagulation parameters of sepsis patients were investigated. Eighty-one patients with sepsis who were admitted to the ICU department of the General Hospital of Ningxia Medical University from April 1, 2015 to December 31, 2015 were collected. Clinical data of the patients were collected. Data were compared using 5 grouping methods: i) the 81 patients were divided into the sepsis group $(n=45)$ and sepsis shock group $(n=36)$; ii) patients were divided into two groups: group A (APACHE II score $\leq 13$, $\mathrm{n}=51$ ); group B (APACHE II score $>13, \mathrm{n}=30$ ); iii) according to Disseminated Intravascular Coagulation Diagnosis Integral System (CDSS), patients were divided into non-disseminated intravascular coagulation (DIC) group (CDSS $<7$ points) and DIC group (CDSS $\geq 7$ points); TEG indexes were compared between the two groups; iv) correlation between TEG indexes and Sequential Organ Failure Assessment (SOFA) scores was analyzed; v) patients were divided into survival group and nonsurvival group and correlations between TEG indicators and prognosis were analyzed. At $6 \mathrm{~h}$ after ICU entry, compared with sepsis group, $\mathrm{R}$ value and $\mathrm{K}$ time were significantly increased, LY30 was also increased, while MA value, coagulation index (CI), and $\alpha$ angle were significantly decreased in the septic shock group $(\mathrm{P}<0.05)$. At $6 \mathrm{~h}$ after ICU entry, compared with sepsis group, $\mathrm{R}$ value and $\mathrm{K}$ time were significantly increased, while MA value, CI, and $\alpha$ angle were significantly decreased in the septic shock group $(\mathrm{P}<0.05)$. Compared with the non-DIC group, the DIC group had prolonged $\mathrm{K}$ time, decreased $\alpha$ angle, increased $\mathrm{R}$ value, and decreased $\mathrm{CI}$ and MA value $(\mathrm{P}<0.05)$. With increase of SOFA scores, $\mathrm{R}$ value and $\mathrm{K}$ value increased significantly, and $\alpha$ angle, MA value,
\end{abstract}

Correspondence to: Dr Xigang Ma, Department of Intensive Care Unit, General Hospital of Ningxia Medical University, 804 Shengli South Street, Yinchuan, Ningxia 750004, P.R. China

E-mail: nyfyicu@163.com

*Contributed equally

Key words: sepsis, thromboelastography, scoring, disseminated intravascular coagulation, prognosis and $\mathrm{CI}$ decreased significantly $(\mathrm{P}<0.05)$. According to $\mathrm{TEG}$, platelet function and fibrinogen function of DIC patients were significantly reduced, and the body showed hypocoagulability.

\section{Introduction}

Sepsis refers to an unregulated host response caused by an infection, resulting in life-threatening organ dysfunction. Conditions of sepsis usually progress rapidly and the prognosis is poor. Sepsis is the main cause of death in ICU (1). Abnormal coagulation system is considered as one of the important mechanisms of the occurrence and development of sepsis and multiple organ dysfunction syndrome (2). With the progression of sepsis, the presence of coagulopathy and coagulation dysfunction is an independent factor affecting the outcome of the disease (3). Abnormality of various coagulation function indicators such as D-dimer, platelet count (PLT), prothrombin time (PT), activated partial thromboplastin time (APTT), and fibrinogen (FIB) was closely correlated with the severity of sepsis (4).

Thromboelastography (TEG) is a simple, cytological-based coagulation test that has the advantages of short measurement time, low blood use, and unaffected heparin. TEG can trace the coagulation process of the body as a form of a graph, which can more intuitively reflect the interaction of various substances in the blood coagulation process (5). TEG reflects the whole process from the beginning of coagulation to the dissolution of blood clots through $\mathrm{R}$ values, $\mathrm{K}$ value, Angle, MA, comprehensive coagulation index (CI) and LY30. Those factors reflect changes in clotting factors, platelets, fibrin, and fibrinolysis at various stages of coagulation. As a result of the effect, the overall functional status of all substances participating in the clotting process is monitored (6). Studies have confirmed that the MA value in TEG testing is a comprehensive assessment of the quantity and function of FIB and platelets involved in blood clot formation and can effectively reflect the functional status of platelets $(7,8)$. Both the $\alpha$-angle and K-time are indicators of FIB function, so the changes in FIB function can be analyzed based on $\alpha$-angle and K-time. Previous findings have shown that monitoring patients' coagulation parameters and TEG parameters can help determine the prognosis and severity of the disease (9). TEG is easy to operate and the graphic is easy to understand. The schematic diagram and diagnostic tree of the TEG are shown in Figs. 1 and 2. 
Findings have shown that TEG can provide a more comprehensive assessment of coagulation status in patients with sepsis and can more sensitively detect abnormal coagulation (10). Coagulation disorders in patients with sepsis are complex and variable, disseminated intravascular coagulation (DIC) may occur and the prognosis is poor. However, DIC is an intermediate link not an independent disease in the complex pathological process of many diseases. Clinical manifestations of DIC are complex and very diverse. Basic diseases and clinical manifestations are the basis for the diagnosis of DIC. Any single laboratory index used for DIC diagnosis has significant limitations. It has been shown that TEG can identify hypocoagulable states in patients with severe sepsis and hypercoagulative states in non-DIC patients with severe sepsis (11). In addition, the maximum amplitude (MA) of thromboelastometry in patients with severe sepsis in ICU stayed constant for several days, and the MA of hypocoagulable states independently predicted 28-day mortality (12). In patients with severe sepsis, changes in TEG variables suggest that progressively worsening hypocoagulation is associated with the risk of death and bleeding (13).

At present, the Acute Physiology, Age and Chronic Health Evaluation (APACHE II) system II is the most widely used method for assessing the condition and prognosis of critically ill patients. APACHE II scores can be used to assess the severity of the disease and has a certain value for prediction of prognosis and mortality risk $(14,15)$. Higher APACHE II scores indicate heavier disease, worse prognosis and higher mortality rate (16).

Previous findings have shown that $(17,18)$, APACHE II score and Sequential Organ Failure Assessment (SOFA) can be used to effectively evaluate the prognosis of critically ill patients, and those factors are closely correlated. With the update of the definition of sepsis (Sepsis-3), the concept of quick Sequential Organ Failure Assessment (qSOFA) and systemic inflammatory response syndrome (SIRS) have been proposed. However, SOFA remains the most reliable method for the diagnosis of sepsis (19). Furthermore, the accuracy of predicting in-hospital mortality of SOFA is higher than that of SIRS or qSOFA scores when adult patients with suspected infections in ICU showed a SOFA score $\geq 2$ (20).

For this study, clinical data of 81 patients with sepsis were collected. The patients were grouped and TEG data were compared to observe changes in coagulation function. The purpose of this study was to investigate the correlation between TEG indicators and the severity of sepsis and provide a theoretical basis for the early detection and treatment of coagulopathy.

\section{Patients and methods}

Patients. The present study used a prospective research method to collect clinical data of coagulation in 81 patients with sepsis who were admitted to the Department of Intensive Care, General Hospital of Ningxia Medical University (Yinchuan, China) from April 1, 2015 to December 31, 2015. Inclusion criteria were: i) ICU sepsis patients admitted to General Hospital of Ningxia Medical University from April, 2015 to December, 2015; ii) conformity to the diagnostic criteria for sepsis and septic shock (refer to 2016 International Guidelines for Treatment of Sepsis and Septic Shock) (21); iii) age $\geq 18$ years. Exclusion criteria: i) patients with incomplete records of medical records; ii) patients with acute cardiovascular and cerebrovascular diseases, liver cirrhosis, congenital coagulation disorder, organ transplantation, blood system diseases, hypersplenism or chronic renal insufficiency or patients needing kidney replacement therapy, long-term radiotherapy and chemotherapy.

The study was approved by the Ethics Committee of General Hospital of Ningxia Medical University. Signed informed consent was obtained from the patients or the guardians.

Grouping method. According to the 2016 International Guidelines for Treatment of Sepsis and Septic Shock, patients were divided into sepsis and septic shock groups (diagnostic criteria are shown in Fig. 3). TEG indicators of the two groups at the time of ICU entry and $6 \mathrm{~h}$ after ICU entry were compared. According to the median APACHE II score within $24 \mathrm{~h}$ after ICU admission, the patients were divided into two groups: group A (APACHE II score $\leq 13, n=51$ ); group B (APACHE II score $>13, n=30$ ), TEG indicators were compared between the two groups. According to the Disseminated Intravascular Coagulation Diagnosis Integral System (CDSS), the patients were divided into non-DIC group (CDSS $<7$ points) and DIC group (CDSS $\geq 7$ points), and TEG indexes were compared between the two groups. Correlations between TEG indexes and SOFA scores of 81 patients at the time of ICU entry were analyzed. Patients were divided into survival group and non-survival group and correlations between TEG indicators and prognosis were analyzed.

Survey contents (using self-made questionnaires to collect case data for statistical analysis). General conditions: patient's age, sex, presence of underlying disease, number of ICU stays, total days of hospitalization, diagnosis, and prognosis. Monitoring indicators: coagulation function (PT, APTT, FIB, TT, FDP, DD, 3P test) and thrombo-elastogram parameters (R, $\mathrm{K}, \mathrm{MA}, \alpha, \mathrm{CI}), \mathrm{LY} 30$ value and PLT at the time of admission to the ICU and $6 \mathrm{~h}$ after admission. According to the diagnostic criteria of sepsis and septic shock group APACHE II scoring was performed within $24 \mathrm{~h}$. Grouping was performed according to median APACHE II score. DIC scores were calculated according to CDSS and grouped according to DIC scores. SOFA score and TEG index grouping and prognostic grouping were also performed.

Normal reference values. Routine coagulation monitoring: APTT, 27.6-34.3 sec; TT, 10.3-16.6 sec; PT, 9.9-12.8 sec; PTA, 70-130\%; FIB, 2.0-4.6 g/l; DD, <1.5 mg/l, FDP, 0-5 $\mu \mathrm{g} / \mathrm{ml}$. Blood routine: PLT, 100-300x10\%/1; PCT, 0.114-0.282\%; PLCR, 13-43\%; MPV, 7.6-13.2 fl; PDW, 9.0-17.0 fl. Thromboplasty index: $\mathrm{R}$ value, 5-10 $\mathrm{min}$; MA value, $50-70 \mathrm{~mm}$; $\alpha$ angle, 53-72 deg; K time, 1-3 min; CI, -3 to 3; LY30, -3-4.0\%.

Statistical analysis. SPSS 17.0 statistical software (SPSS, Inc., Chicago, IL, USA) was used to process all the data. Normal distribution data were expressed as mean \pm standard deviation. Mann-Whitney test was used for comparison between groups. Pearson rank was used for correlation analysis between two variables. $\mathrm{P}<0.05$ was considered to indicate a statistically significant difference. 


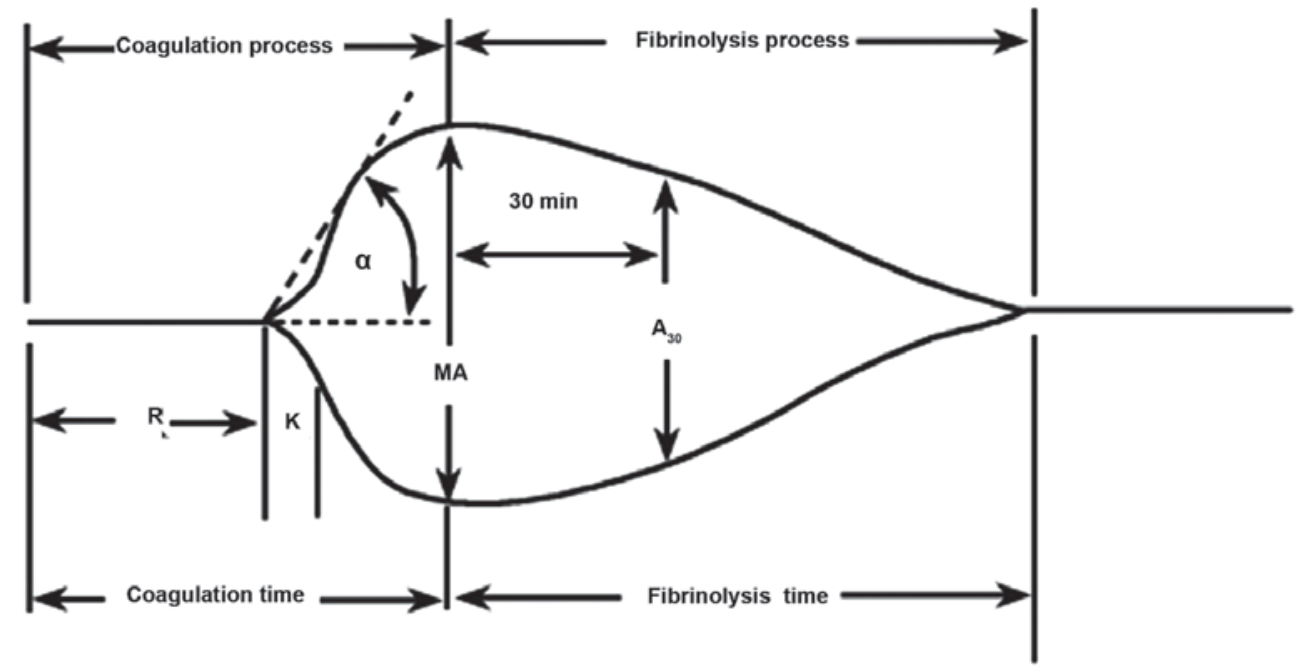

Figure 1. Diagram of TEG. TEG, thromboelastography.

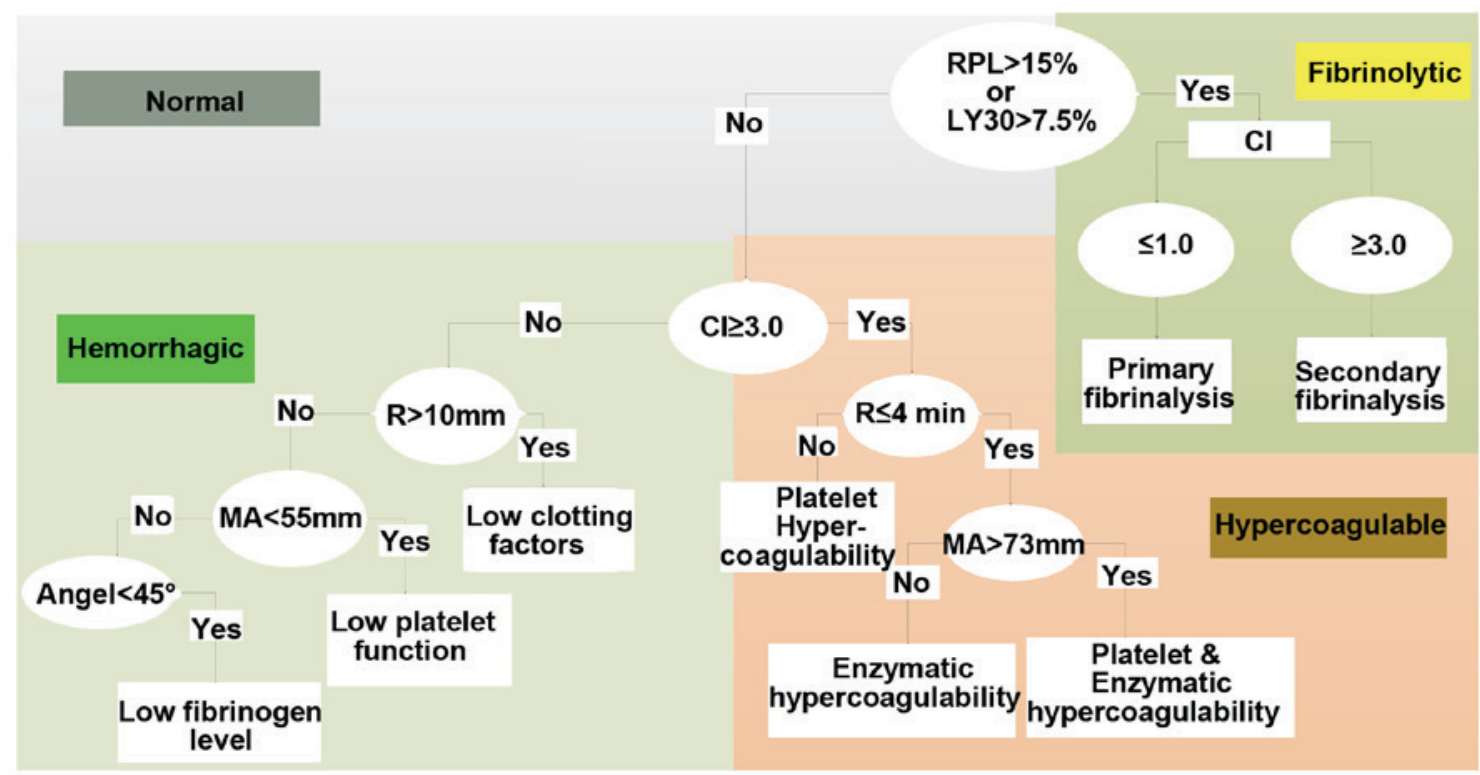

Figure 2. Diagnostic tree of the TEG. R time, refers to the time from the start to the amplitude of $2 \mathrm{~mm}$ in TEG image. The R time reflects the process of coagulation initiation. The prolonged $\mathrm{R}$ value represents the lack of clotting factor in the body, indicating a hypocoagulable state, and conversely indicating a hypercoagulable state in the body. $\mathrm{K}$ time, refers to the time between the amplitude of 2 and $20 \mathrm{~mm}$ in the TEG image. K time reflects the rate of blood clot formation and is an indicator of FIB function. The prolongation of $\mathrm{K}$ time suggested that the function of FIB was reduced, and on the contrary, it meant that FIB was hyperfunctioning. $\alpha$ angle is the slope from $\mathrm{R}$ to $\mathrm{K}$ in the TEG image. $\mathrm{K}$ time and $\alpha$ angle reflect the common effect of fibrin and platelets on the beginning of clot formation. The MA value reflects the maximum hardness or strength of the clot formation. The main manifestation is the platelet aggregation function, which can also reflect the stability of the thrombus. The increase of MA value indicates that the platelet function is hyperactive, and vice versa is the reduced platelet function. $\mathrm{CI}$ is a comprehensive coagulation that reflects the overall state of coagulation. Normal value of CI is between -3 and 3; CI <-3 indicates hypocoagulable state, and conversely suggests a hypercoagulable state. LY30 refers to the percentage of fibrinolysis 30 min after the clot reached maximum intensity and indicates the rate of fibrinolysis. TEG, thromboelastography; FIB, fibrinogen; MA, maximum amplitude; CI, coagulation index.

\section{Results}

Patients. Age range of the 81 sepsis patients was 19-84 years and the average age was $59.14 \pm 13.42$ years. The patients included 52 males $(64.2 \%)$ and 29 females (35.8\%). There were no significant differences in the patient's sex, age and other basic data among sepsis group, APACHE II score group, DIC group, TEG index and SOFA score correlation group and prognosis group (Tables I-III).
Comparison of TEG parameters between patients with sepsis and septic shock. At the time of ICU entry, compared with sepsis group, $\mathrm{K}$ time in septic shock group was significantly longer than that of sepsis group (Fig. 4B), and the angle $\alpha$, MA value and CI decreased significantly (Fig. 4C,D,F), while LY30 increased significantly (Fig. 4E). After 6 h, ICU entry, compared with sepsis group, $\mathrm{R}$ value and $\mathrm{K}$ time of the septic shock group were significantly prolonged (Fig. 4A and B), while the MA value, $\mathrm{CI}$, and $\alpha$ angle were significantly 


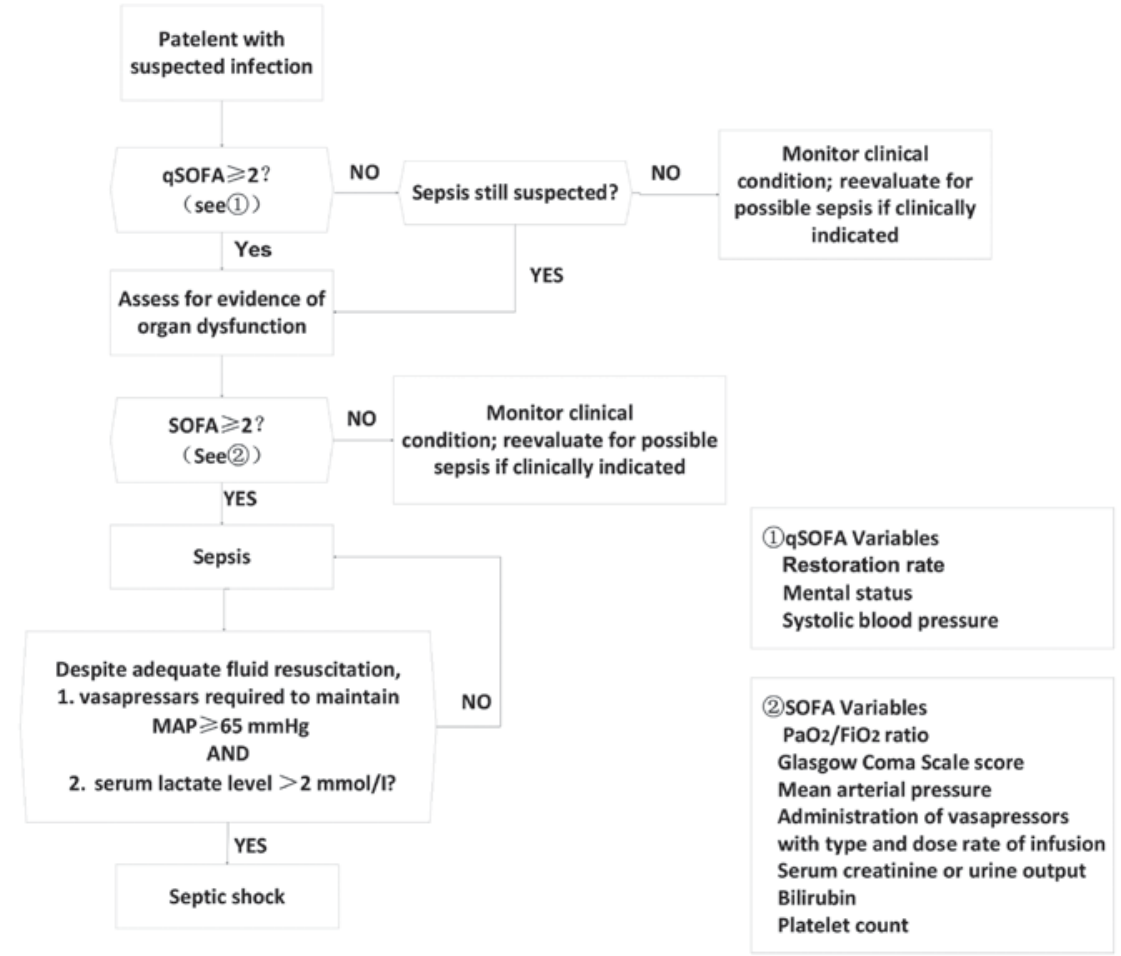

Figure 3. Diagnostic criteria for sepsis and septic shock. SOFA, Sequential Organ Failure Assessment.

Table I. Sepsis groups (mean \pm standard deviation).

\begin{tabular}{lcc}
\hline Basic data & $\begin{array}{c}\text { Sepsis group } \\
(\mathrm{n}=45)\end{array}$ & $\begin{array}{c}\text { Septic shock group } \\
(\mathrm{n}=36)\end{array}$ \\
\hline Age & $58.71 \pm 12.36$ & $59.67 \pm 14.78$ \\
Sex & 31 males & 21 males \\
& 14 females & 15 females \\
APACHE II score & $10.7 \pm 0.5$ & $17.2 \pm 5.9$ \\
\hline
\end{tabular}

APACHE, Acute Physiology, Age and Chronic Health Evaluation.

Table II. APACHE II score groups (mean \pm standard deviation).

\begin{tabular}{lcc}
\hline Basic data & Group A $(\mathrm{n}=51)$ & Group B $(\mathrm{n}=30)$ \\
\hline Age & $56.18 \pm 13.14$ & $64.17 \pm 12.55$ \\
Sex & 33 males & 19 males \\
& 18 females & 11 females
\end{tabular}

APACHE, Acute Physiology, Age and Chronic Health Evaluation.

decreased (Fig. 4C,D,F), and LY30 was significantly increased (Fig. 4E).

APACHE II score grouping and TEG index comparison

TEG test indicators at ICU entry. Compared with group A, group B had longer $\mathrm{K}$ value, lower MA value, lower CI index
Table III. DIC and non-DIC groups (mean \pm standard deviation).

\begin{tabular}{lcc}
\hline Basic data & $\begin{array}{c}\text { Non-DIC group } \\
(\mathrm{n}=64)\end{array}$ & $\begin{array}{c}\text { DIC group } \\
(\mathrm{n}=17)\end{array}$ \\
\hline Age & $59.70 \pm 13.17$ & $57.00 \pm 14.55$ \\
Sex & 37 males & 15 females \\
& 27 females & 2 females \\
\hline
\end{tabular}

DIC, disseminated intravascular coagulation.

Table IV. APACHE II score grouping and TEG index ( \pm standard deviation) at admission (mean \pm standard deviation).

\begin{tabular}{lccl}
\hline TEG indexes & Group A & Group B & P-value \\
\hline R value (min) & $6.39 \pm 2.57$ & $9.54 \pm 4.90$ & 0.087 \\
K value (min) & $2.60 \pm 1.48$ & $3.79 \pm 2.94$ & $0.013^{\mathrm{a}}$ \\
$\alpha$ angle (deg) & $56.18 \pm 13.36$ & $44.01 \pm 16.75$ & 0.055 \\
MA value (mm) & $60.59 \pm 9.69$ & $54.14 \pm 13.07$ & $0.022^{\mathrm{a}}$ \\
CI & $-1.29 \pm 3.66$ & $-5.61 \pm 6.45$ & $0.019^{\mathrm{a}}$ \\
LY30 (\%) & $0.79 \pm 2.24$ & $0.37 \pm 0.57$ & $0.030^{\mathrm{a}}$ \\
\hline
\end{tabular}

Compared with group A, group B had prolonged $\mathrm{K}$ value, decreased MA value and CI and lower LY30 ( $\mathrm{P}<0.05),\left({ }^{\mathrm{a}} \mathrm{P}<0.05\right)$. APACHE, Acute Physiology, Age and Chronic Health Evaluation; TEG, thromboelastography; MA, maximum amplitude; CI, coagulation index.

and lower LY30 $(\mathrm{P}<0.05)$. TEG test indicators at ICU entry are shown in Table IV. 
A
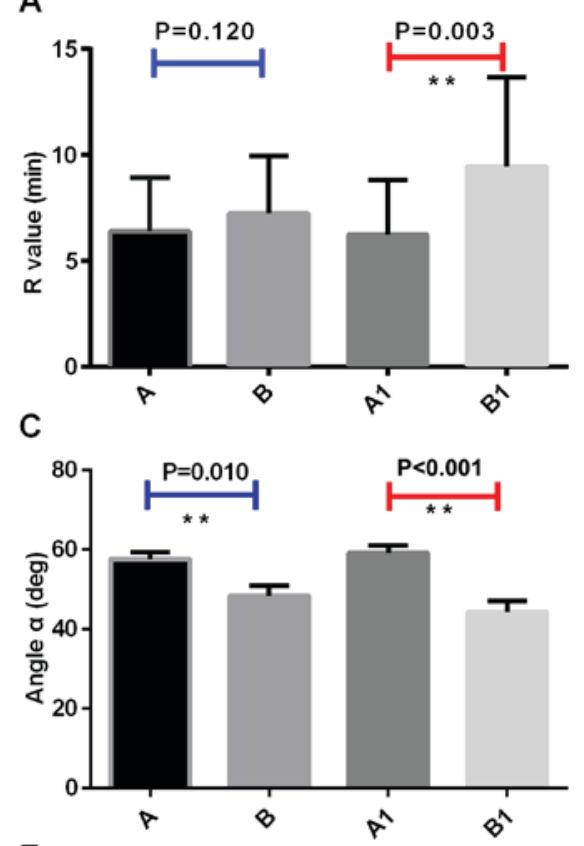

$\mathrm{E}$

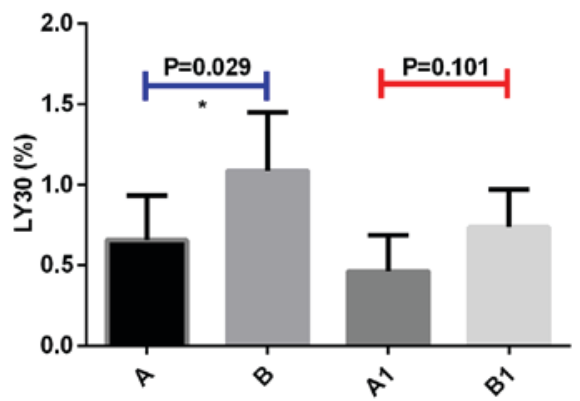

B

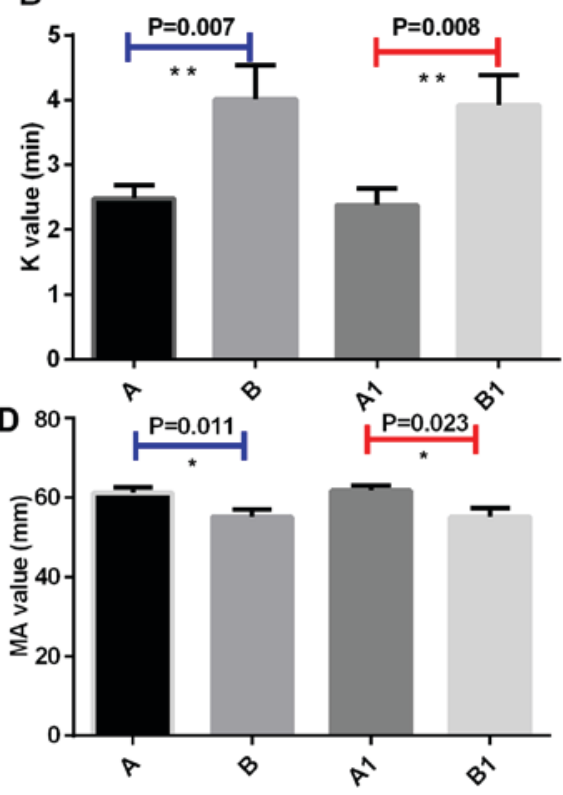

$F$

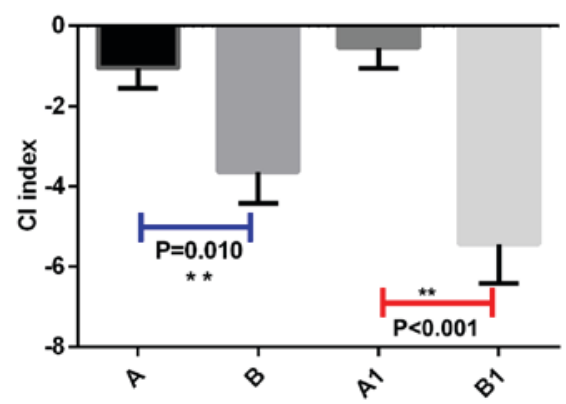

Figure 4. Comparison of TEG detection parameters between sepsis and septic shock groups. TEG test indicators for patients with sepsis in ICU; TEG test indicators for the patients with septic shock in ICU. A1, TEG indicators of sepsis patients in ICU at $6 \mathrm{~h}$ after admission. B1, TEG indicators of septic shock patients in ICU at $6 \mathrm{~h}$ after admission. (A) R value, (B) K time, (C) $\alpha$ angle, (D) MA value, (E) LY30, and (F) CI ( ${ }^{*} \mathrm{P} \leq 0.05$; $\left.{ }^{* *} \mathrm{P} \leq 0.01\right)$. TEG, thromboelastography; MA, maximum amplitude; CI, coagulation index.

Table V. APACHE II score grouping and TEG index at $6 \mathrm{~h}$ after admission (mean \pm standard deviation).

\begin{tabular}{lccc}
\hline TEG indexes & Group A & Group B & P-value \\
\hline R value (min) & $6.31 \pm 2.56$ & $8.29 \pm 4.26$ & $0.017^{\mathrm{a}}$ \\
K value (min) & $2.36 \pm 1.70$ & $3.54 \pm 2.66$ & $0.035^{\mathrm{a}}$ \\
$\alpha$ angle (deg) & $59.20 \pm 12.33$ & $47.98 \pm 17.17$ & $<0.001^{\mathrm{b}}$ \\
MA value (mm) & $60.65 \pm 8.68$ & $56.9 \pm 12.75$ & $0.021^{\mathrm{a}}$ \\
CI & $-0.90 \pm 3.53$ & $-3.60 \pm 6.11$ & $0.001^{\mathrm{b}}$ \\
LY30 (\%) & $3.59 \pm 15.13$ & $3.52 \pm 10.89$ & 0.172
\end{tabular}

APACHE, Acute Physiology, Age and Chronic Health Evaluation; TEG, thromboelastography; MA, maximum amplitude; CI, coagulation index. ${ }^{\mathrm{a}} \mathrm{P} \leq 0.05 ;{ }^{\mathrm{b}} \mathrm{P} \leq 0.01$.

TEG test indicators at $6 \mathrm{~h}$ after ICU entry (Table V). Compared with group A, R value was prolonged, CI decreased, MA value decreased, $\mathrm{K}$ time was prolonged and $\alpha$ angle decreased in group $\mathrm{B}(\mathrm{P} \leq 0.05 ; \mathrm{P} \leq 0.01)$.
Table VI. DIC grouping and TEG indicators (mean \pm standard deviation).

\begin{tabular}{lccc}
\hline $\begin{array}{l}\text { Coagulation } \\
\text { indicators }\end{array}$ & $\begin{array}{c}\text { Non-DIC group } \\
(\mathrm{n}=65)\end{array}$ & $\begin{array}{c}\text { DIC group } \\
(\mathrm{n}=16)\end{array}$ & P-value \\
\hline MA value (mm) & $60.69 \pm 8.92$ & $49.24 \pm 12.1$ & $0.001^{\mathrm{b}}$ \\
K value (min) & $2.78 \pm 1.63$ & $4.73 \pm 4.08$ & $0.017^{\mathrm{b}}$ \\
R value (min) & $6.51 \pm 2.65$ & $7.83 \pm 2.36$ & $0.036^{\mathrm{a}}$ \\
$\alpha$ angle (deg) & $55.46 \pm 13.84$ & $45.77 \pm 14.58$ & $0.025^{\mathrm{a}}$ \\
CI & $-1.46 \pm 3.70$ & $-5.17 \pm 5.08$ & $0.006^{\mathrm{b}}$ \\
LY30 (\%) & $0.64 \pm 1.73$ & $1.70 \pm 2.78$ & 0.056 \\
\hline
\end{tabular}

Compared with the non-DIC group, $\mathrm{R}$ and $\mathrm{K}$ times was prolonged, $\mathrm{CI}$ and MA indexes decreased and $\alpha$ angle decreased $(\mathrm{P}<0.05)$. ( ${ }^{\mathrm{P}} \mathrm{P} \leq 0.05$; $\left.{ }^{\mathrm{b}} \mathrm{P} \leq 0.01\right)$. DIC, disseminated intravascular coagulation; TEG, thromboelastography; MA, maximum amplitude; CI, coagulation index.

Comparison of DIC grouping and TEG indicators. The comparison of DIC grouping and TEG indicators is shown 

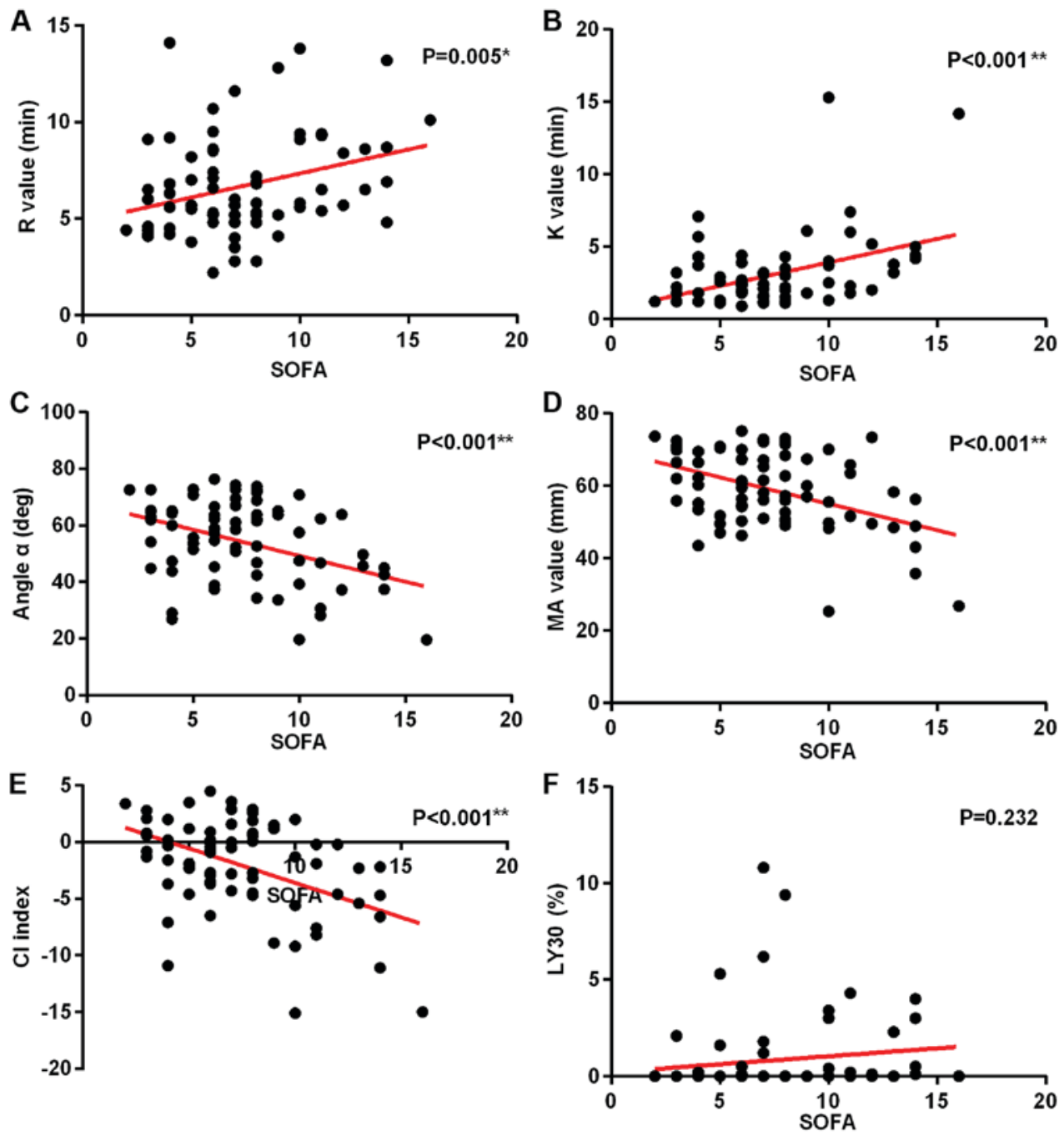

Figure 5. Correlation analysis of SOFA scores and TEG indicators. (A) R values, (B) K value, (C) $\alpha$ angle, (D) MA value, (E) CI indicators, and (F) LY30. $\left(\right.$ ( $\left.\mathrm{P} \leq 0.05 ;{ }^{* *} \mathrm{P} \leq 0.01\right)$. SOFA, Sequential Organ Failure Assessment; TEG, thromboelastography; MA, maximum amplitude; CI, coagulation index.

Table VII. Prognosis and TEG index (mean \pm standard deviation).

\begin{tabular}{lccc}
\hline Coagulation index & Survival & Death & P-value \\
\hline MA value (mm) & $58.48 \pm 10.40$ & $58.20 \pm 11.78$ & 0.841 \\
K value (min) & $3.12 \pm 2.24$ & $3.33 \pm 3.20$ & 0.937 \\
R value (min) & $6.59 \pm 2.42$ & $7.57 \pm 3.38$ & 0.301 \\
$\alpha$ angle (deg) & $53.26 \pm 14.29$ & $57.77 \pm 15.45$ & 0.640 \\
CI & $-2.08 \pm 4.06$ & $-2.69 \pm 5.13$ & 0.733 \\
LY30 (\%) & $0.91 \pm 2.15$ & $0.57 \pm 1.22$ & 0.851 \\
MA 1 (mm) & $15.63 \pm 4.03$ & $58.49 \pm 13.36$ & 0.715 \\
K 1 (min) & $3.10 \pm 2.41$ & $2.87 \pm 2.47$ & 0.480 \\
R 1 (min) & $7.46 \pm 3.80$ & $8.57 \pm 3.56$ & 0.118 \\
$\alpha$ angle (deg) & $52.84 \pm 16.54$ & $51.35 \pm 16.58$ & 0.711 \\
CI & $-2.50 \pm 5.31$ & $-3.64 \pm 5.31$ & 0.359 \\
LY30 1 $(\%)$ & $0.46 \pm 1.14$ & $1.15 \pm 2.41$ & 0.118
\end{tabular}

No statistical significance was found between prognostic groups in TEG indicators at the time of ICU entry and $6 \mathrm{~h}$ after ICU entry. TEG, thromboelastography; MA, maximum amplitude; CI, coagulation index. in Table VI. The results showed that compared with non-DIC group, the median $\mathrm{R}$ value of TEG was prolonged, and CI index was decreased in DIC group, indicating that the body was in a state of hypocoagulation. MA value was decreased, indicating that platelet function was decreased. K time was prolonged, and $\alpha$ angle was decreased, suggesting that fibrinogen function was decreased.

Correlation analysis between SOFA scores and TEG indicators. With the increase of SOFA scores, $\mathrm{R}$ value and $\mathrm{K}$ time increased significantly (Fig. 5A and B), while $\alpha$ angle, MA value, and CI decreased significantly (Fig. 5C-E). Fig. 5F shows that there is no significant correlation between SOFA score and LY30. In this group, a correlation study of PLTs and TEG indicators was also performed. It was found that with the decrease of PLT, R value, K value, and LY30 decreased significantly (Fig. 6A,B,F), while $\alpha$ angle, and MA value and $\mathrm{CI}$ increased significantly (Fig. 6C-E) $(\mathrm{P}<0.05)$.

Prognostic groups and TEG indicators. Prognostic groups and TEG indicators are shown in Table VII. No statistical significance was found between prognostic groups in TEG indicators at the time of ICU entry and $6 \mathrm{~h}$ after ICU entry. 

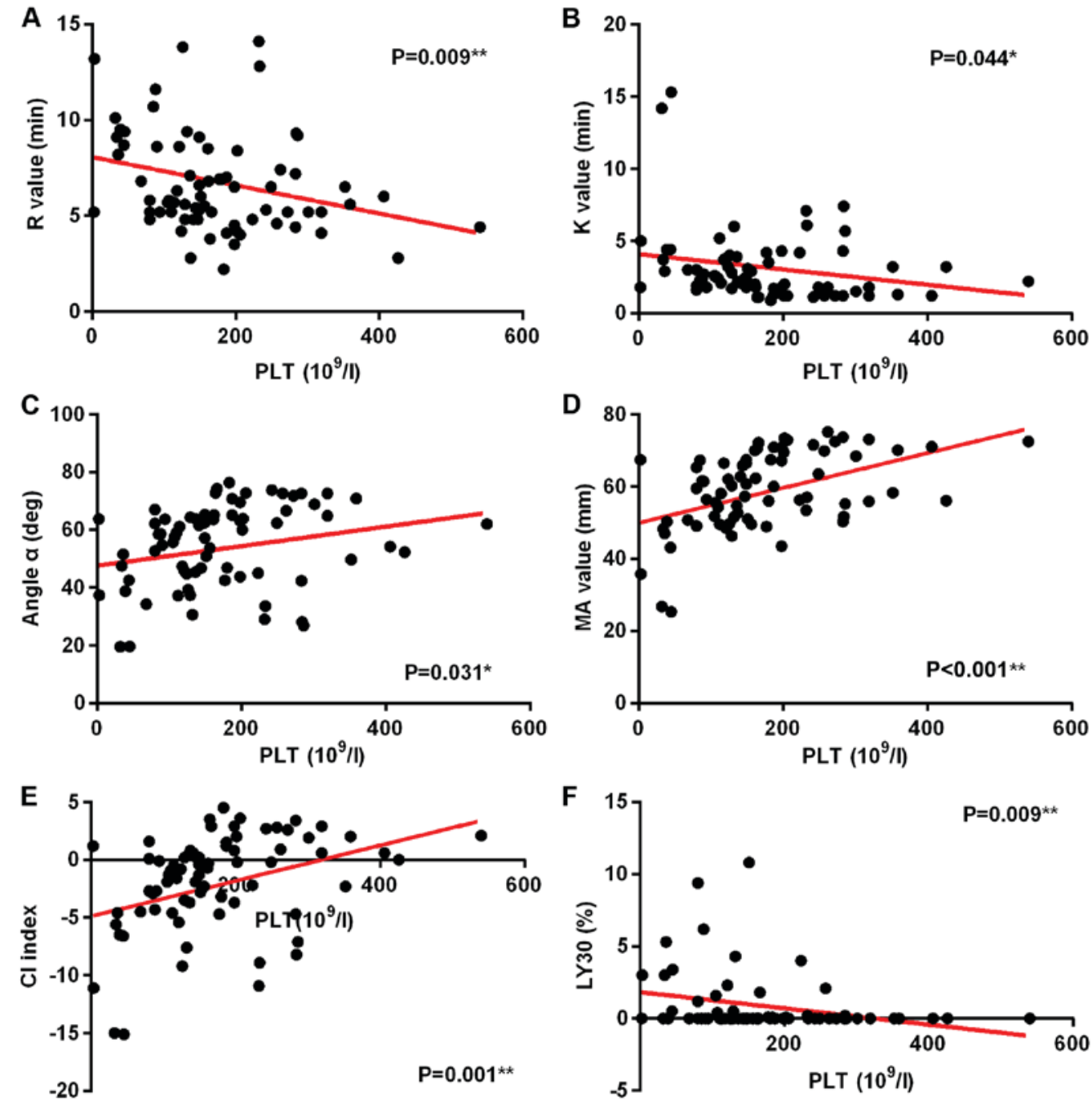

Figure 6. Correlation analysis of PLT and TEG indicators. (A) R value, (B) K time, (C) $\alpha$ angle, (D) MA value, (E) CI indicators, and (F) LY30. ("P $\leq 0.05$; $\left.{ }^{* *} \mathrm{P} \leq 0.01\right)$. PLT, platelet count; TEG, thromboelastography; MA, maximum amplitude; CI, coagulation index.

\section{Discussion}

Sepsis is the leading cause of death in critically ill patients worldwide. Pathogenesis of this disease remains unclear and efforts are being made on the diagnosis and treatment of this disease. An increasing number of studies have shown that the activation of the coagulation system and microcirculatory dysfunction play a very important role in the occurrence and development of sepsis. It has been shown that coagulation disorder is persistent throughout sepsis and is an important factor for prognosis (22). Findings of the present study suggest that the coagulation disorders in patients with sepsis main have the following mechanisms: i) tissue factor induces the production of thrombin through an exogenous coagulation pathway, thus FIB is activated and converted into fibrin, and platelet activation aggregates (23); ii) impaired systemic physiological anticoagulant system, such as the lack of activated protein C, antithrombin III, and tissue factor pathway inhibitors, causes downregulation of anticoagulation (24); iii) disturbance of the fibrinolytic system occurs, and the concentration of plasminogen activator inhibitor-I in plasma increases, resulting in the closure of the fibrinolytic system (10), which leads to decreased fibrinolysis. Activation of the coagulation system and the subsequent downregulation contribute to the disorder of coagulation in patients with sepsis. Inflammatory response interacts with disturbance of coagulation to cause organ dysfunction. Therefore, timely monitoring of coagulation changes and early treatment of coagulopathy can block the deterioration of sepsis. At present, the coagulation function monitoring methods commonly used in clinical practice include traditional coagulation function testing and TEG tests. Traditional coagulation test can only evaluate part of the clotting system and cannot truly reflect the blood coagulation balance in the body. TEG has obvious advantages in reflecting the effects of plasma components and cellular components on blood clotting at different stages of coagulation, assessing the overall blood coagulation, and determining coagulation factor function.

The TEG test can convert the kinetic process of coagulation to a tracing curve, which can more intuitively reflect the interaction of various substances in the coagulation process (25). Changes in clotting factors, platelets, fibrin, and fibrinolysis at various stages of coagulation, as well as the state of the substances involved in the coagulation process, can all be well reflected by the TEG test (7). The main parameters of the TEG test include coagulation reaction time ( $\mathrm{R}$ value), CI, MA, 
coagulation rate ( $\alpha$ angle), and kinetics of clot development ( $\mathrm{K}$ values). TEG has been widely used clinically, especially in cardiovascular surgery, organ transplant surgery, blood transfusion, anticoagulation and antiplatelet therapy.

The current study shows that an increase of severity of disease is accompanied by an increase of APACHE II score. The extent of coagulation disorders is positively correlated with the severity of the disease. In this study, 81 patients were divided into different groups according to five grouping method.

Based on the 2016 International Guidelines for the Treatment of Sepsis and Septic Shock, patients were divided into sepsis group and septic shock group, and TEG indicator at the time of ICU entry and $6 \mathrm{~h}$ after ICU entry were compared. At the time of ICU entry, compared with sepsis group, K time in septic shock group was significantly longer than that of sepsis group, and the angle $\alpha$, MA value and CI decreased significantly, while LY30 increased significantly, indicating that patients in septic shock group exhibited hypocoagulable states, platelet and FIB functions were reduced, and fibrinolysis progressed. At $6 \mathrm{~h}$ after ICU entry, compared with sepsis group, $\mathrm{R}$ value and $\mathrm{K}$ time of the septic shock group were significantly prolonged, while the MA value, $\mathrm{CI}$, and $\alpha$ angle were significantly decreased, and LY30 was significantly increased, indicating that patients in the shock group showed hypocoagulation, and platelet function and FIB function were significantly reduced. This set of results shows that the degree of disorder in coagulation function of patients is positively correlated with the severity of sepsis. When sepsis occurs, bacteria and endotoxins stimulate vascular endothelial cells, activate the release of various cytokines, coagulation factors, and vasoactive substances, activate platelet aggregation, and condense TXB2 and cytokines, leading to tissue ischemia, hypoxia, the formation of micro-thrombosis, damage to the vascular endothelium, inhibited anticoagulation system and fibrinolytic system, microcirculation disorders, and ultimately the development of septic shock (26). Results obtained in this study are in accordance with the law of occurrence and development of coagulation in sepsis. The specific coagulation status reflected in TEG is prolongation of $\mathrm{K}$ and the decrease of $\alpha$ angle suggested a decrease in FIB function, a decrease in MA value suggested a decrease in platelet function, and a decrease in CI suggested a tendency toward hypocoagulability. This reflects the complete picture of coagulation, which is also an advantage of TEG. Traditional coagulation function test does not reflect the status of platelet and FIB function, but only reflects the quantitative changes. Results of this group study showed that the more severe the condition of patients with sepsis, the greater the degree of coagulation disorders. Clinical monitoring of coagulation function, and timely and effective treatment may block or delay the occurrence and development of sepsis.

APACHE II score as an internationally recognized system for evaluating the condition of critically ill patients can be used to perform more comprehensive assessments of the severity of the illness (27). The higher the APACHE II score is, the more severe the patient condition is and the higher the mortality rate will be. On the contrary, the lower the APACHE II score is, the less severe the patient's condition is, and the better the prognosis of such a patient is (28). Therefore,
APACHE II score is positively related to the severity and mortality of the disease (29), so it is widely used clinically. The second grouping method in this study was based on the median APACHE II score. Eighty-one patients were divided into group A (APACHE II score $\leq 13$ points) and group B (APACHE II score $>13$ points), and statistical analysis was performed on TEG scores at the time of ICU entry and $6 \mathrm{~h}$ after ICU entry. At the time of ICU entry, compared with group A, the K time was significantly prolonged, MA value, and $\mathrm{CI}$ were significantly decreased in group B. At $6 \mathrm{~h}$ after ICU entry, compared with sepsis group, $\mathrm{R}$ value and $\mathrm{K}$ time were significantly increased, while MA value, $\mathrm{CI}$ and $\alpha$ angle were significantly decreased in septic shock group. These data suggest that as the APACHE II score increases, the body's hypocoagulable state becomes more pronounced, and fibrin and platelet function declines. This shows that the severity of the disease is positively correlated with the APACHE II score, and as the disease progresses, the extent of coagulation disorders worsens. In this process, TEG test can reflect whether there is consumption of coagulation factors and whether the function of clotting factors is reduced, thereby more fully reflecting the overall coagulation state of the patient.

Coagulation dysfunction in patients with sepsis is complicated and changeable, and there are many influencing factors. Patients with sepsis are prone to DIC and, in the event of DIC, the prognosis is usually poor. However, DIC is not an independent disease, but is an intermediate link in the pathological process of multiple complex diseases. This dynamic change of process is rapid, clinical symptoms are hidden, and the evaluation ability of diagnostic indicators is limited, which brings great difficulties to the clinical identification of DIC. Primary disease and clinical manifestations are the basis of the diagnosis of DIC. On this basis, laboratory indicators need to be comprehensively evaluated. Any single laboratory index used to evaluate the process of coagulation and diagnosis of DIC has great limitations (30-33). Gold standard for the diagnosis of DIC is lacking. In recent years, experts from Japan, Europe, and the United States have successively proposed multi-indicator DIC integral diagnostic systems, those systems mainly include Japanese Society of Emergency Medicine system, the Japanese Ministry of Health and Welfare system, and the International Thrombosis and Hemostasis Association system. However, the accuracy and practicality of these three standards are still widely controversial. The scope of current diagnostic scoring system, inclusion criteria, and the boundary value classification of indicators are still open to question, and accurate diagnosis of DIC is still a difficult task.

In 2017, the Chinese Medical Association Hematology Branch Thrombosis and Hemostasis Group established the China Disseminated Intravascular Coagulation Diagnosis Score System (CDSS) through a multi-center, large-scale retrospective and prospective study, making the DIC diagnostic criteria more in line with conditions in China. The third grouping method in this study was to collect laboratory indicators of 81 patients when they entered the ICU. According to the CDSS, they were divided into non-DIC group (CDSS $<7$ points) and DIC group (CDSS $\geq 7$ points). TEG indicators were compared and results showed that compared with the non-DIC group, R time was prolonged and CI decreased in DIC group suggesting a hypocoagulable state. 
Besides, decreased MA values suggested decreased platelet function and prolonged $\mathrm{K}$ time and decreased $\alpha$ angle suggest FIB dysfunction. As known, the current CIs required for the diagnosis of DIC are mainly PT, APTT, PLT, FIB, FDP and D-dimer. These are quantitative indicators and do not reflect the functional status of clotting factors, but TEG detection can reflect the function of clotting factors. TEG showed a decrease in platelet function and FIB function in DIC group, and it indicated that the body showed a hypocoagulable state, which was not possible with traditional coagulation parameters. However, this does not mean that the TEG test can replace the traditional coagulation function test. In clinical work, traditional blood coagulation testing methods can be combined with TEG testing to comprehensively evaluate the patient's coagulation status, thereby assisting diagnosis and guiding treatment.

The newly proposed Sepsis-3 concept leads to the emergence of qSOFA concept. qSOFA can help to quickly determine the severity of the patient's condition, and it is easier to get qSOFA score in the clinic. However, SOFA score may be more accurate in reflecting the severity of sepsis. SOFA score can be used to assess the evolution of functional impairment in various organ systems. The higher the total score is, the greater the number of organ system failures are, which indicates that more severe condition and higher mortality rate. SOFA scoring system is mainly based on six systems including respiratory system, nervous system, circulatory system, liver function, coagulation system and kidney function, among which the evaluation of coagulation system is based on PLT, and there is no traditional CI and TEG index available for the evaluation of coagulation system. It is well known that the coagulation state of patients with sepsis is extremely complicated and changeable. It is impossible to represent coagulation function by a certain CI, but a comprehensive analysis of the condition and test results is required to evaluate coagulation function. A number of studies have shown that there is a correlation between TEG index and the PLT (34-36). The same conclusion was made in this study (Fig. 6). Therefore, it is proposed to analyze the relationship between the TEG index and the SOFA score to analyze the coagulation status of patients with sepsis. The fourth grouping method of this study was to collect the SOFA scores of 81 patients and the TEG index at the time of admission. The correlation between SOFA scores and TEG parameters was analyzed. Results showed that, as the score of SOFA increases, $\mathrm{R}$ value increases, and CI decreases significantly, suggesting that the body exhibits a hypocoagulable state; $K$ value increases significantly and angle of $\alpha$ decreases, suggesting a decrease in FIB function; and the decrease in MA value indicates reduced function of platelet. Evaluation of the coagulation system by the SOFA score is based on the number of platelets. This study found that number of platelets was closely correlated with TEG indicators. Therefore, it can be concluded that TEG can reflect the functional status of clotting factors in patients with sepsis, as well as the overall blood coagulation, and it is in accordance with the occurrence and development of sepsis. It also shows that TEG can be used to determine severity of sepsis.

Eighty-one patients were divided into the survival and death groups. Statistical analysis was performed on the TEG indicators at the time of admission and $6 \mathrm{~h}$ after admission. No correlation was found between the TEG indicators and the prognosis. The consideration may be associated with the sample size. In addition, the pathogenesis of sepsis is extremely complicated and remains unclear. Coagulation dysfunction is one of the factors in the occurrence and development of sepsis, and its own mechanism is also very complicated and affected by many factors. Therefore, a single index may not directly reflect the prognosis of patients with sepsis.

In conclusion, coagulation disorders in patients with sepsis have been confirmed, but the mechanism is still not fully understood. Indicators in TEG testing, such as $\mathrm{R}$ value, $\mathrm{K}$ time, $\alpha$ angle, MA value and CI, can help determine the severity of the patient's condition. Although the advantage of TEG in detecting the function of the coagulation system is more prominent, it still cannot replace the traditional coagulation test and PLT test. It is necessary to combine these three methods to make a comprehensive analysis and accurately reflect the coagulation status of patients with sepsis, thereby achieving timely and effective treatment. Close attention should be paid on changes in the blood coagulation system in patients with sepsis. Traditional coagulation tests, routine blood tests, and TEG tests should be combined to comprehensively determine coagulation status in patients with sepsis, so as to make early, comprehensive, accurate, and objective diagnosis.

The patient experienced obvious hypocoagulable state, FIB function and platelet function decline according to TEG, from the sepsis to septic shock process, and those conditions became worse with the increase of the severity of disease. Moreover, platelet function and FIB function of DIC patients were significantly reduced, and the body showed hypocoagulability.

\section{Acknowledgements}

Not applicable.

\section{Funding}

This study was supported by the funding project of Ningxia Medical University Fund for Scientific Research (no. XM2015054, no. XM2016027), Ningxia Natural Science Foundation (no. NZ17151) and the regional project of National Science Foundation of China (no. 81260583).

\section{Availability of data and materials}

The datasets used and/or analyzed during the present study are available from the corresponding author on reasonable request.

\section{Authors' contributions}

WenyanZ and WenjieZ collected and analyzed the general information of patients. JB recorded coagulation function indicators. SM analyzed thrombo-elastogram parameters. QL and XM interpreted normal reference values. All authors read and approved the final manuscript.

\section{Ethics approval and consent to participate}

The study was approved by the Ethics Committee of General Hospital of Ningxia Medical University (Yinchuan, China). 
Signed informed consent was obtained from the patients or the guardians.

\section{Patient consent for publication}

Not applicable.

\section{Competing interests}

The authors declare that they have no competing interests.

\section{References}

1. Azkárate I, Sebastián R, Cabarcos E, Choperena G, Pascal M and Salas E: A prospective, observational severe sepsis/septic shock registry in a tertiary hospital in the province of Guipuzcoa (Spain). Med Intensiva 36: 250-256, 2012 (In Spanish).

2. Samuels JM, Moore HB and Moore EE: Coagulopathy in severe sepsis: Interconnectivity of coagulation and the immune system. Surg Infect (Larchmt) 19: 208-215, 2018.

3. Wang Y, Ouyang Y, Liu B, Ma X and Ding R: Platelet activation and antiplatelet therapy in sepsis: A narrative review. Thromb Res 166: 28-36, 2018.

4. Wang Y, Wang D, Fu J and Liu Y: Predictive value of SOFA, qSOFA score and traditional evaluation index on sepsis prognosis. Zhonghua Wei Zhong Bing Ji Jiu Yi Xue 29: 700-704, 2017 (In Chinese)

5. Wang L, Bastarache JA and Ware LB: The coagulation cascade in sepsis. Curr Pharm Des 14: 1860-1869, 2008.

6. Reikvam H, Steien E, Hauge B, Liseth K, Hagen KG, Størkson R and Hervig T: Thrombelastography. Transfus Apheresis Sci 40: $119-123,2009$.

7. Kozek-Langenecker S: Management of massive operative blood loss. Minerva Anestesiol 73: 401-415, 2007.

8. Salooja N and Perry DJ: Thrombelastography. Blood Coagul Fibrinolysis 12: 327-337, 2001.

9. Kunio NR, Differding JA, Watson KM, Stucke RS and Schreiber MA: Thrombelastography-identified coagulopathy is associated with increased morbidity and mortality after traumatic brain injury. Am J Surg 203: 584-588, 2012.

10. Ren J, Zhao Y, Yuan Y, Han G, Li W, Huang Q, Tong Z and Li J: Complement depletion deteriorates clinical outcomes of severe abdominal sepsis: A conspirator of infection and coagulopathy in crime? PLoS One 7: e47095, 2012.

11. Sivula M, Pettilä V, Niemi TT, Varpula M and Kuitunen AH: Thromboelastometry in patients with severe sepsis and disseminated intravascular coagulation. Blood Coagul Fibrinolysis 20 419-426, 2009

12. Ostrowski SR, Windeløv NA, Ibsen M, Haase N, Perner A and Johansson PI: Consecutive thrombelastography clot strength profiles in patients with severe sepsis and their association with 28-day mortality: A prospective study. J Crit Care 28: 317.e1-317. e11, 2013.

13. Haase N, Ostrowski SR, Wetterslev J, Lange T, Møller MH, Tousi H, Steensen M, Pott F, Søe-Jensen P, Nielsen J, et al: Thromboelastography in patients with severe sepsis: A prospective cohort study. Intensive Care Med 41: 77-85, 2015.

14. Braber A and van Zanten AR: Unravelling post-ICU mortality: Predictors and causes of death. Eur J Anaesthesiol 27: 486-490, 2010.

15. Namendys-Silva SA, Texcocano-Becerra J and Herrera-Gómez A: Prognostic factors in critically ill patients with solid tumours admitted to an oncological intensive care unit. Anaesth Intensive Care 38: 317-324, 2010.

16. Jianmin Q, Xueliang Y, Liqin L, Yongsheng W, Licang H and Yuanxin $\mathrm{H}$ : Value of continuous video EEG and EEG responses to thermesthesia stimulation in prognosis evaluation of comatose patients after cardiopulmonary resuscitation. Open Med (Wars) 13: 35-40, 2018.

17. Singh RK, Poddar B, Baronia AK, Azim A, Gurjar M, Singhal S, Srivastava S and Saigal S: Audit of patients with severe acute pancreatitis admitted to an intensive care unit. Indian J Gastroenterol 31: 243-252, 2012.

18. Qiao Q, Lu G, Li M, Shen Y and Xu D: Prediction of outcome in critically ill elderly patients using APACHE II and SOFA scores. J Int Med Res 40: 1114-1121, 2012.
19. Goulden R, Hoyle MC, Monis J, Railton D, Riley V, Martin P, Martina R and Nsutebu E: qSOFA, SIRS and NEWS for predicting inhospital mortality and ICU admission in emergency admissions treated as sepsis. Emerg Med J 35: 345-349, 2018.

20. Mato A, Fuchs BD, Heitjan DF, Mick R, Halpern SD, Shah PD, Jacobs S, Olson EM, Schuster SJ, Ujjani C, et al: Utility of the systemic inflammatory response syndrome (SIRS) criteria in predicting the onset of septic shock in hospitalized patients with hematologic malignancies. Cancer Biol Ther 8: 1095-1100, 2009.

21. Rhodes A, Evans LE, Alhazzani W, Levy MM, Antonelli M, Ferrer R, Kumar A, Sevransky JE, Sprung CL, Nunnally ME, et al: Surviving Sepsis Campaign: International Guidelines for Management of Sepsis and Septic Shock: 2016. Crit Care Med 45: 486-552, 2017.

22. Knoebl P: Blood coagulation disorders in septic patients. Wien Med Wochenschr 160: 129-138, 2010.

23. Levi M and Schultz M: The inflammation-coagulation axis as an important intermediate pathway in acute lung injury. Crit Care 12: 144-147, 2008.

24. Lissalde-Lavigne G, Combescure C, Muller L, Bengler C, Raillard A, Lefrant JY and Gris JC: Simple coagulation tests improve survival prediction in patients with septic shock. J Thromb Haemost 6: 645-653, 2008.

25. Luddington RJ: Thrombelastography/thromboelastometry. Clin Lab Haematol 27: 81-90, 2005.

26. Nakae H, Endo S, Inada K, Takakuwa T, Kasai T and Yoshida M: Relationship between thromboxane B2 and 6-keto-prostaglandin F1 alpha in sepsis. Res Commun Chem Pathol Pharmacol 83: 297-302, 1994.

27. Alarcón G, Fardella P, Conte G, Vargas V, Parada X and Cuneo M: Changes in coagulation in patients with sepsis. Rev Med Chil 121: 537-541, 1993 (In Spanish).

28. Wang H, Li Z, Yin M, Chen XM, Ding SF, Li C, Zhai Q, Li Y, Liu $\mathrm{H}$ and Wu DW: Combination of Acute Physiology and Chronic Health Evaluation II score, early lactate area, and $\mathrm{N}$-terminal prohormone of brain natriuretic peptide levels as a predictor of mortality in geriatric patients with septic shock. J Crit Care 30: 304-309, 2015.

29. Chen FG and Koh KF: Septic shock in a surgical intensive care - validation of multiorgan and APACHE II scores in predicting outcome. Ann Acad Med Singapore 23: 447-451, 1994.

30. Levi M and Scully M: How I treat disseminated intravascular coagulation. Blood 131: 845-854, 2018.

31. Di Nisio M, Baudo F, Cosmi B, D'Angelo A, De Gasperi A, Malato A, Schiavoni M and Squizzato A; Italian Society for Thrombosis and Haemostasis: Diagnosis and treatment of disseminated intravascular coagulation: Guidelines of the Italian Society for Haemostasis and Thrombosis (SISET). Thromb Res 129: e177-e184, 2012.

32. Louw SJ, Mayne ALH and Mayne ES: Evaluation of the diagnostic utility of individual parameters in the disseminated intravascular coagulation (DIC) panel for use in under resourced settings. Int J Lab Hematol 40: e46-e48, 2018.

33. Masuda T, Shoko T and Deguchi Y: Clinical investigation of coagulation markers for early detection of sepsis-induced disseminated intravascular coagulation: A single-center, prospective observational study. Clin Appl Thromb Hemost 24: 1082-1087, 2018

34. Dorman BH, Spinale FG, Bailey MK, Kratz JM and Roy RC: Identification of patients at risk for excessive blood loss during coronary artery bypass surgery: Thromboelastography versus coagulation screen. Anesth Analg 76: 694-700, 1993.

35. Rocha LL, Pessoa CM, Neto AS, do Prado RR, Silva E, de Almeida MD, Correa TD; POCKET Trial Investigators: Thromboelastometry versus standard coagulation tests versus restrictive protocol to guide blood transfusion prior to central venous catheterization in cirrhosis: Study protocol for a randomized controlled trial. Trials 18: 85, 2017.

36. Bowbrick VA, Mikhailidis DP and Stansby G: Influence of platelet count and activity on thromboelastography parameters. Platelets 14: 219-224, 2003. International (CC BY-NC-ND 4.0) License. 